\title{
Developing a strategy to promote the generation and effective use of population health research for NSW Health: 2011-2015
}

\author{
Janice S. Biggs ${ }^{\mathrm{A}}$ and Beth Stickney ${ }^{\mathrm{B}, \mathrm{C}}$ \\ ${ }^{\mathrm{A}}$ NSW Public Health Officer Training Program, \\ NSW Department of Health \\ ${ }^{\mathrm{B}}$ Centre for Epidemiology and Research, \\ $N S W$ Department of Health \\ ${ }^{\mathrm{C}}$ Corresponding author.Email: bstic@doh.health.nsw.gov.au
}

\begin{abstract}
The Population Health Division of the NSW Department of Health has developed a 5 -year strategy to improve the effectiveness of its resource investment in population health research. This paper describes the development of the strategy, Promoting the generation and effective use of population health research in NSW: a Strategy for NSW Health 2011-2015. A review of Australian and international strategic research documents and stakeholder interviews was conducted to support the development of the strategy. The findings from these two processes influenced the structure of the document and supported the inclusion of strategies and actions to assist with identifying research priorities, improving communication, enhancing networks and partnerships, supporting workforce development initiatives, providing research infrastructure, enhancing research and the use of research evidence and streamlining research governance and ethics processes. Small group discussions and a detailed review of literature were conducted to refine the thinking around four of the more complex aspects of the strategy. Finally, a broad consultation process was used to test the face validity of the proposed strategy content.
\end{abstract}

The value of using evidence to inform health policy and practice is widely acknowledged. ${ }^{1,2}$ A number of models have been developed; these processes are complex and evaluations of strategies to increase the use of evidence in policies and programs are rare. ${ }^{3,4}$
NSW Health invests in population health research through both the Population Health Division of the New South Wales (NSW) Department of Health and local population health services. The Population Health Division has recently developed a strategy which outlines how it will facilitate the conduct of high-quality, relevant, population health research and the use of research evidence in policy and practice in NSW Health. This paper describes how this strategy, Promoting the generation and effective use of population health research in NSW: a Strategy for NSW Health 2011-2015 (the Strategy), was developed.

\section{Methods}

An Advisory Committee, comprising senior population health managers from the NSW Department of Health and local health services, academics in population health from NSW universities and senior managers from relevant non-government organisations, was established to provide guidance on the development and content of the Strategy.

To inform development three projects were undertaken: (1) a review of strategic documents that support decisionmaking for health research; (2) a series of stakeholder interviews; and (3) a rapid review of the literature that examined strategies to increase the use of evidence from research in population health policy and programs. The first two projects, the review of strategic documents and the stakeholder interviews, form the basis of this paper. ${ }^{5,6}$ The findings from the rapid literature review have been reported elsewhere. ${ }^{4}$ Three small-group discussions were held to refine complex aspects of the Strategy and, as a final step, the draft Strategy was circulated for broad consultation.

\section{Review of strategic documents}

A review of strategic health research documents was conducted by searching the websites of health departments of Australian states and territories and of other countries that have similar health care structures to Australia. Web searches were conducted using the search terms 'strategic directions', 'framework', 'plan', 'public health research', 'population health research' and 'health care research'. Documents were considered eligible for inclusion if they: were from comparable countries; related to public health, population health or health research; and were recent 
enough to be relevant to the NSW Strategy. Policy officers from the health departments of other jurisdictions were contacted to confirm whether they had a research plan and to ensure that current documents, including those from the grey literature, were included in the review.

Based on a preliminary analysis of the documents, an outline for the Strategy was prepared which covered advocacy for, the planning of, conducting, building capacity for, and using, population health research. The outline also proposed the inclusion of guidelines for researchers and users of research.

A detailed analysis of all the documents gathered was then conducted: their structure, content and terminology was summarised and systematically coded using the proposed headings in the Strategy outline. Additional themes not included in the outline were identified. The findings from this review, and input from the Advisory Committee, informed the second version of the Strategy outline that was used in the stakeholder interviews. This second Strategy outline also contained a draft vision, aim, objectives and key strategies.

\section{Stakeholder interviews}

A purposive selection process was used to include stakeholder groups in the interview process. ${ }^{7}$ Twelve people were selected for individual interviews based upon their role (policy maker, health service manager or researcher, at state or local level). Three group interviews were held, two with NSW Department of Health managers and one with senior population health representatives from NSW universities. Ethical approval for all interviews was obtained through the University of New South Wales and all participants signed a consent form prior to interview.

Each interview took between 30 and 60 minutes. Information was collected using a combination of non-directive and standardised open-ended questions. Questions focused on participants' experience of conducting and using research, structures and strategies to support research and its use in population health, and gathering feedback on the Strategy outline.

Themes from the interviews were coded by two investigators based on the propositions in the Strategy outline. ${ }^{7}$ Emerging themes and recurring patterns of interest were also identified and coded. ${ }^{8}$ Iterative analysis was used; that is, the data were examined, coded and compared until saturation was reached. In consultation with the Advisory Committee, the results of the stakeholder interviews and the rapid review of strategies to increase the use of evidence from research in population health policy and programs ${ }^{4}$ were used to develop a draft Strategy document, including a modified vision, aim, objectives and strategies, and with the inclusion of detailed actions to achieve the objectives.
Further group discussions were held to refine the sections on research priority setting, workforce development and fostering supportive organisational cultures.

\section{Final consultation on the draft Strategy}

The draft Strategy was circulated widely among a broad range of population health stakeholders in NSW (including those from the NSW Department of Health, local population health services, universities and non-government organisations) to confirm the face validity of the content of the Strategy. Limited modifications were made as a result of this final consultation.

\section{Results}

\section{Review of strategic documents}

The fifteen documents identified in the review had different formats: over half were strategies, five were plans, two were frameworks and one was a policy statement. ${ }^{9-23}$ Although the terminology used differed, the strategies and plans shared similar structures and included specific actions to achieve identified goals and objectives. The strategies were longer-term (e.g. 5 years), higher-level and had substantial implementation budgets. The plans were more operational and covered shorter time periods. The frameworks were promotional in nature and the policy statement identified issues and methods of implementing solutions. Only one document included guidelines (for research funders).

Most of the actions within the documents reflected the proposed content of the NSW Strategy. Similar themes included: identifying research priorities to focus research planning; workforce development and research infrastructure to build research capacity; and enhancing the use of research evidence in policy and practice through networks and partnerships. The approach to identifying research priorities varied from linking to state health priorities or prescribing priority research questions, to assessing priorities against set criteria and providing principles of effective priority setting. Additional themes included research leadership (not well elucidated in the documents), and research governance and ethics. None of the documents contained specific advocacy strategies.

\section{Stakeholder interviews}

Four major themes emerged from the analysis of the stakeholder interviews.

\section{Theme 1 - Improving communication and sharing of information}

A common issue was the need for improved communication about: research developments; funding opportunities; infrastructure assets and training opportunities. Communication across four dimensions was identified as 
important: (1) from the NSW Department of Health to local health services, universities and external organisations; (2) between researchers; (3) between researchers and policy makers and practitioners; and (4) across the Centres in the Population Health Division.

Many participants referred to the value of formal networks for formulating and conducting research, seeking advice, offering encouragement, sharing and disseminating information, fostering collaborations, and identifying gaps in research.

Decision makers noted that time constraints precluded conducting comprehensive literature searches to inform policy or practice decisions. A facility that summarises and stores research findings in an easy-to-digest format was suggested. Reviews conducted by NSW Healthfunded research centres were valued, but thought not to be widely known.

Increasing access to, and utilisation of, existing data sets was recommended to enhance a coordinated, cohesive approach to research within NSW.

Those external to the Department often heard about Departmental funding through informal networks. There was a general consensus that funding processes could be more consistent and transparent and that potential synergies between Centres in the Population Health Division regarding funding procedures should be explored.

\section{Theme 2 - Developing partnerships}

Strengthening partnerships was seen as an important element of the NSW Strategy, underpinning many of the other strategies in the document. Long-term programmatic engagement between researchers and policy makers and practitioners was seen as essential to enhance the quality and relevance of population health research in NSW and for effective use of evidence in policy and practice. Partnerships supporting joint research projects between NSW Health and local universities were highlighted as beneficial and desirable, however, challenges to development of these partnerships were also raised.

Research partnerships were seen as a way for local health services to conduct larger research projects, thus ensuring sufficient size, power and effect, and to provide support for less experienced researchers. Partnerships were also perceived to foster common understanding between NSW Health, affiliated organisations and local universities.

\section{Theme 3 - Workforce development}

Support and encouragement for conducting population health research varied considerably across NSW Health. Practitioners said they often had to conduct research alongside their full-time 'day-to-day activities' and felt that research should be legitimised as part of the work of the Population Health Division and local population health services. The need for supportive policy and practice environments that value and use research evidence was emphasised.

Capacity building for several key groups in NSW was identified as important to improve population health research and its use, for example: NSW Health researchers at the state and local level require technical skills such as in mixed and complex research methods, biostatistics and epidemiology; policy makers and the population health workforce require broad research literacy to be able to use research evidence and consider appropriate evaluation techniques; and university researchers require support to undertake policy relevant research. Building collaborations with local universities and promoting opportunities to be involved in larger projects were identified as strategies for developing research skills.

Fellowships and scholarships were identified as valuable, cost-effective ways to: increase researcher capacity; strengthen links between NSW Health and universities; foster relevant research; and build the capacity of organisations to secure research funds. Mentorship for skills development and confidence building was not raised explicitly, but was implied through comments such as needing 'support from other staff' and 'someone to go to'.

Theme 4 - Enhancing research and the use of research evidence

Population health research priorities were seen as an essential component of the Strategy to enhance the relevance of research and the use of research evidence. As research priorities will change over time, participants recommended the establishment of processes for identifying, updating and communicating priorities for NSW Health, rather than specifying priorities in the document.

Simplifying ethics procedures, particularly for low and negligible risk projects, was cited as a way to streamline research processes. Guidance on research governance was also sought, particularly in relation to accessing datasets and developing research partnerships.

Using knowledge brokers and establishing long-term, project-based relationships were acknowledged as approaches for bringing researchers, policy makers and practitioners together and assisting with processes of exchange and knowledge co-creation to support the use of research evidence in practice.

Comments from the final consultation and small group discussions of complex issues informed the final version of the Strategy document, summarised in Figure 1 and Table 1. 
Vision

Aim which will lead to better population health and reduced health inequities in NSW

$\uparrow$

Objectives

Increase research that is relevant to NSW Health population health policy and practice Improve the quality of population health research in NSW Health

Increase the use of research evidence in NSW Health population health policies and practice

Strengthen population health research capability in NSW Health

\begin{tabular}{l|l|}
\cline { 2 - 3 } Key strategies & $\begin{array}{l}\text { S1. Facilitate the generation of high } \\
\text { quality research that is relevant to } \\
\text { population health policy and practice } \\
\text { S1.1: Identify and ensure a focus on } \\
\text { NSW Health priorities for population } \\
\text { health research } \\
\text { S1.2: Fund and commission research } \\
\text { S1.3: Plan and undertake research }\end{array}$ \\
\end{tabular}

S1. Facilitate the generation of high
quality research that is relevant to population health policy and practice

S1.1: Identify and ensure a focus on NSW Health priorities for population health research

S1.2: Fund and commission research

\section{S2. Maximise the use of research to improve population health and reduce health inequities}

S2.1: Facilitate synthesis of and access to research evidence

S2.2: Develop policy and practice environments that value and use research evidence

S2.3: Foster research environments that promote the use of research evidence in policy and practice

\section{S3. Build population health research} capability

S3.1: Develop and maintain the population health research workforce

S3.2: Develop and maintain population health research infrastructure

S3.3: Foster links and partnerships for collaborative population health research

Figure 1. The Population Health Research Strategy framework.

\section{Discussion}

The review of strategic documents and stakeholder interviews contributed to shaping the structure and content of the NSW Strategy. The review confirmed that structuring the document as a longer-term strategy with specific actions was appropriate.

Encouragingly, the review endorsed the suggested content and focus of the Strategy, however some modifications were indicated. In the reviewed documents guidelines for research were rare, so for brevity a decision was made to remove guidelines from the NSW Strategy. Research governance and ethics, a common inclusion in the documents, was added to the Strategy while advocacy was removed as a separate action. Further, the review elucidated the multiple approaches used in other jurisdictions for identifying population health research priorities.

The stakeholder interviews were used to test the proposed content of the Strategy, a process recommended by Bridgeman and Davis (2004). ${ }^{24}$ In particular, the importance of improved communication and information sharing was highlighted and is supported in the literature. . $25,26^{2}$ Actions have been included in the Strategy to keep researchers, policy makers and practitioners informed of: population health research priorities; research funding opportunities; funded research and its use in policy and practice; research syntheses; networks and training opportunities; data sets; infrastructure assets; and emerging research developments from within the Population Health Division.

Stakeholders frequently referred to the benefits of partnerships for research and the use of research evidence, a common theme in reviews of health research. ${ }^{27-29}$ Partnerships were seen to reduce the tendency for services to work in silos, particularly important during a period of health service reform. ${ }^{30}$ Essential to many other strategies, partnerships were seen to have the strongest connection with building research capability. The Strategy therefore places partnerships within the capability-building frame and focuses on improving researcher-practitioner links, particularly with universities. Effective partnerships are also key inputs and outcomes of many of the other actions in the document.

Underpinning capability for research was an expressed need to legitimise research as a core function of state and local population health services, initially described as a deficit in NSW Research: Prescription for Health. ${ }^{29}$ The Strategy itself will be an initial driver for raising the profile and importance of research in population health services and specific actions have been included to foster environments that support the generation and use of research.

In relation to setting research priorities for population health, the Strategy adopted the approach favoured by 


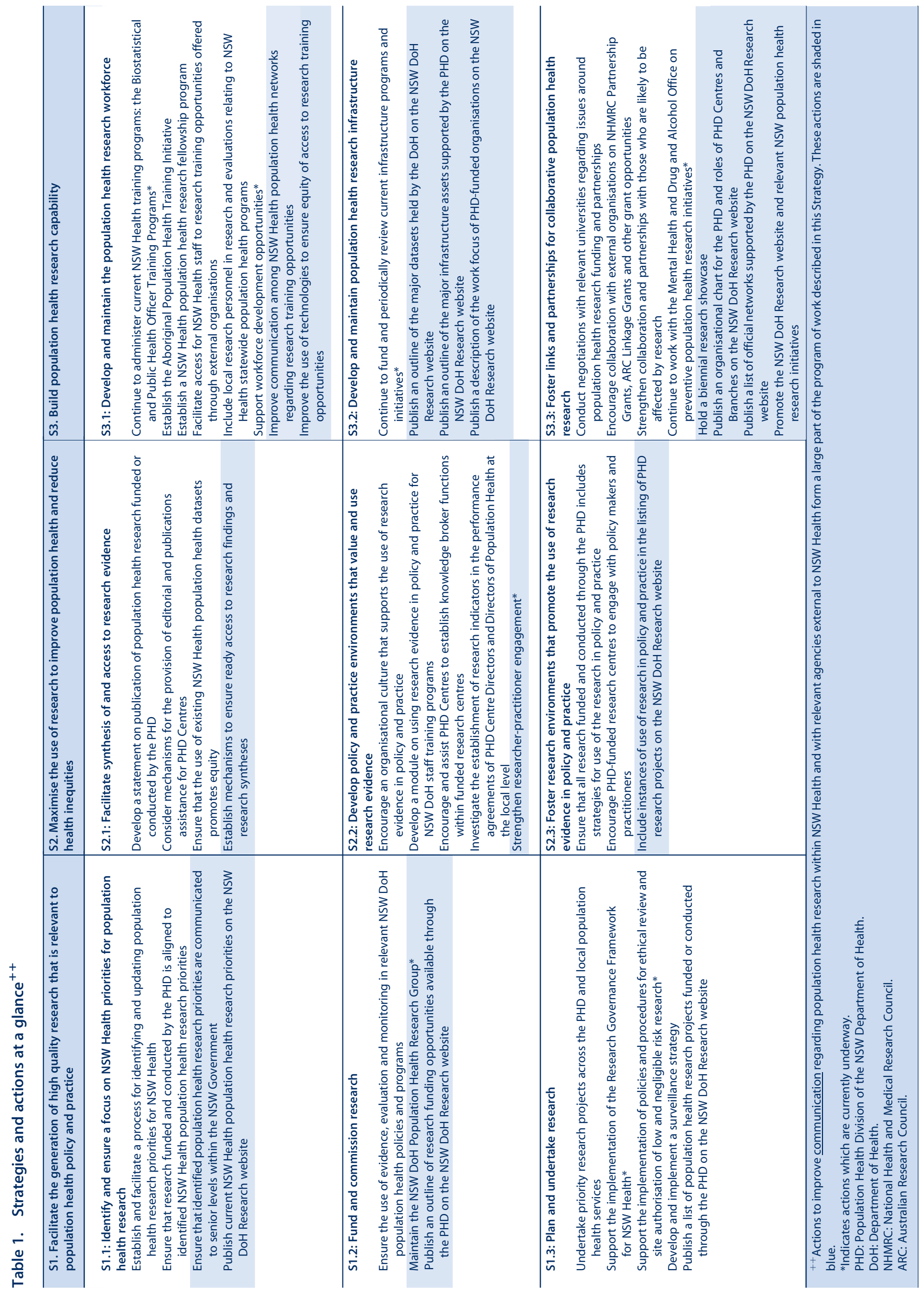


stakeholders, that is, the establishment of a process for identification and dissemination of priorities.

Most of the other actions raised in the interviews have been incorporated into the Strategy and many of these are supported in the literature. For example, the value of knowledge brokerage services, ${ }^{31}$ success of fellowship programs in other jurisdictions, ${ }^{5}$ enhancing the capacity of the existing research workforce through networks and improved access to training opportunities ${ }^{26,32}$ and simplifying ethics procedures. $^{33}$

The actions in the Strategy are designed to be implemented within existing NSW Health funds, with a focus on managing current investment more strategically and working better with internal and external partners.

\section{Conclusion}

Review and stakeholder consultation processes used to formulate NSW Health's Promoting the generation and effective use of population health research in NSW: a Strategy for NSW Health 2011-2015 refined ideas for actions to improve the generation and use of population health research within current budget allocations. These processes strengthened the relevance and comprehensiveness of the Strategy. The Population Health Division will facilitate implementation of the Strategy and report on progress and outcomes.

Promoting the generation and effective use of population health research in NSW: a strategy for NSW Health 2011-2015 is available from: http://www.health.nsw.gov. $\mathrm{au} /$ resources

\section{Acknowledgments}

The authors would like to thank the NSW Health Strategic Directions for Population Health Research Advisory Committee and those involved in the stakeholder interviews, small group discussions and final consultation for their contribution.

\section{References}

1. Banks G. Challenges of Evidence-Based Policy-Making. Australian Government Productivity Commission. Canberra: Commonwealth of Australia; 2009. Available from: http://www. apsc.gov.au/publications09/evidencebasedpolicy.pdf (Cited 4 March 2011.)

2. Productivity Commission. Strengthening Evidence-based Policy in the Australian Federation, Volume 2: Background Paper. Canberra: Productivity Commission; 2010.

3. Kitson A, Rycroft-Malone J, Harvey G, McCormack B, Seers K, Titchen A. Evaluating the successful implementation of evidence into practice using the PARiHS framework: theoretical and practical challenges. Implement Sci 2008; 3: 1. doi:10.1186/ 1748-5908-3-1

4. Moore G, Todd A, Redman S. Strategies to increase the use of evidence from research in population health policy and programs: a rapid review. November 2009. Available from the Centre for Epidemiology and Research, NSW Department of Health.

5. Biggs J. A review of strategic documents that support decisionmaking for population health research. February 2010. Available on request from the Centre for Epidemiology and Research, NSW Department of Health.

6. Biggs J. The results of stakeholder consultations to inform the development of the Strategic Directions for Population Health Research. March 2010. Available on request from the Centre for Epidemiology and Research, NSW Department of Health.

7. Patton MQ. Qualitative research and evaluation methods. 3rd ed. Thousand Oaks, CA: Sage Publications; 2002.

8. Burnard P. A method of analysing interview transcripts in qualitative research. Nurse Educ Today 1991; 11(6): 461-6. doi:10.1016/0260-6917(91)90009-Y

9. Canadian Institutes of Health Research. Health Research Roadmap: Creating innovative research for better health and health care: 2009/10-2013/14; 2009.

10. Government of South Australia, Department of Human Services. Research and Evaluation Framework: 2002-2005.

11. Government of South Australia, Department of Health. Strategic health research program. Research priorities: general health topics; 2007-2008.

12. Government of South Australia, Department of Health. Strategic health research program. Research priorities: health systems research; 2007-2008.

13. Health Protection Agency. Providing the Evidence Base for Public Health: The Health Protection Agency's Research Strategy 2005-10; 2005.

14. Health Research Council of New Zealand. HRC Strategic Plan 2008-2013; 2008.

15. NHS Department of Health, Research and Development Directorate. Best Research for Best Health: A new national health research strategy - the NHS contribution to health research in England; 2006.

16. NHS Department of Health. A Research and Development Strategy for Public Health; 2001.

17. Queensland Health. Queensland Health's Research and Development Plan; 2007-2010.

18. Queensland Health. Research for a healthier future: 2020. Health and Medical Research and Development Strategy; 2009.

19. Saskatchewan Health. Healthy People. A Healthy Province; 2004.

20. Scottish Executive. Prevention/Wellness: Research strategy for Health and Healthcare; 2003.

21. Victorian Government, Department of Human Services. Public Health Research Policy Statement: New knowledge for improved health; 2005.

22. Victorian Health Promotion Foundation. Lead, empower, support, connect. Victorian Health Promotion Foundation: Strategic Priorities; 2006-2009.

23. Welsh Assembly Government, Wales Office of Research and Development for Health and Social Care. A Health and Social Care Research and Development Strategic Framework for Wales: a consultation document; 2002. 
24. Bridgeman P, Davis G. The Australian Policy Handbook. 3rd ed. Sydney: Allen and Unwin; 2004.

25. Straus S, Haynes RB. Managing evidence-based knowledge: the need for reliable, relevant and readable resources. CMAJ 2009; 180(9): 942-5. doi:10.1503/cmaj.081697

26. Van Wave TW, Scrutchfield FD, Honoré PA. Recent Advances in Public Health Systems Research in the United States. Annu Rev Public Health 2010; 31: 283-95. doi:10.1146/annurev. publhealth.012809.103550

27. National Health and Medical Research Council. Report of the Review of Public Health Research Funding in Australia, December 2008. Canberra: Commonwealth of Australia; 2009.

28. National Health and Hospitals Reform Commission. Beyond the Blame Game: Accountability and performance benchmarks for the next Australian Health Care Agreements. Canberra: NHHRC; 2008.
29. NSW Government. NSW Research: a Prescription for Health. Report of a review commissioned by the Minister for Science and Medical Research, the Hon Frank Sartor MP. Sydney: NSW Government; 2004.

30. Australian Government. A National Health and Hospitals Network for Australia's Future. Australian Government; 2010.

31. Redman S, Jorm L, Haines M. Increasing the use of research in health policy: the Sax Institute model. Australasian Epidemiologist 2008; 15(3): 15-8.

32. Madden L, Salmon A. Public health workforce: results of a NSW statewide consultation on the development of the national public health workforce. NS W Public Health Bull 1999; 10(3): 19-21. doi:10.1071/NB99009

33. NSW Department of Health. Improving ethical review and site authorisation processes in NSW: Action Plan. Sydney: NSW Department of Health; 2009. 